\title{
Factores predisponentes para ictericia neonatal en los pacientes egresados de la UCI neonatal, Hospital Infantil los Ángeles de Pasto
}

\author{
Predisposing factors for neonatal jaundice in patients discharged from neonatal ICU, in los Ángeles children's \\ Hospital in Pasto
}

\author{
Angie Lizeth Galíndez-González ${ }^{*}$ orcid.org/0000-0003-4416-5854 \\ Sara Raquel Carrera-Benavides ${ }^{1}$ orcid.org/0000-0002-7871-333X \\ Angely Alejandra Díaz-Jiménez' orcid.org/0000-0002-9304-4357 \\ Mavir Bibiana Martínez-Burbano' ${ }^{1}$ orcid.org/0000-0001-9322-7834
}

1 Programa de Medicina, Fundación Universitaria San Martin. San Juan de Pasto, Colombia

Fecha de recepción: Junio 18 - $2017 \quad$ Fecha de revisión: Septiembre 12 - $2017 \quad$ Fecha de aceptación: Diciembre 1 - 2017

Galíndez-González AL, Carrera-Benavides SR, Díaz-Jiménez AA, Martínez-Burbano MB. Factores predisponentes para ictericia neonatal en los pacientes egresados de la UCI neonatal, Hospital Infantil los Ángeles de Pasto. Univ. Salud. 2017;19(3):352-358. DOI: http://dx.doi.org/10.22267/rus.171903.97

\section{Resumen}

Introducción: La ictericia neonatal es una de las condiciones más frecuentes en los recién nacidos, a pesar de ser benigna, un tratamiento inadecuado puede llegar a ser nocivo para el paciente. Objetivo: Determinar los factores predisponentes de ictericia neonatal en los pacientes egresados de la UCI neonatal del Hospital Infantil Los Ángeles de la ciudad de Pasto (Nariño) en el periodo enero de 2007 - agosto de 2011. Materiales y métodos: Estudio observacional, descriptivo, retrospectivo y cuantitativo. Se estudiaron 608 historias clínicas de neonatos egresados con diagnóstico de ictericia neonatal; las variables estudiadas se analizaron con el complemento de Excel, XLSTATPro 7.5.2. Resultados: Los principales factores predisponentes encontrados en la población, fueron la lactancia materna exclusiva y el género masculino en un $87 \%$, y $57,40 \%$ respectivamente, además $90,79 \%$ fueron recién nacidos a término, 92,93\% tuvieron peso adecuado para la edad gestacional y 54,93\% presentaron ictericia neonatal entre los 2 y 7 días de vida extrauterina. Conclusiones: La ictericia neonatal está asociada a factores maternos y neonatales, tanto modificables como no modificables, que pueden ser abordados con estrategias adecuadas para reducir la carga de enfermedad.

Palabras clave: Ictericia neonatal; factores predisponentes; hiperbilirrubinemia; neonatos. (Fuente: DeCS, Bireme).

\begin{abstract}
Introduction: Neonatal jaundice is one of the most common conditions in newborns; however, improper treatment can be harmful to the patient, despite being benign. Objective: To determine the predisposing factors of neonatal jaundice in patients discharged from the neonatal ICU of Los Angeles children's Hospital in the city of Pasto (Nariño) between January 2007and August 2011. Materials and methods: An observational, descriptive, retrospective and quantitative study was conducted. 608 clinical histories of discharged infants diagnosed with neonatal jaundice were studied. The variables studied were analyzed with the complement of Excel, XLSTAT-Pro 7.5.2. Results: The main predisposing factors found in the population were exclusive breastfeeding and male gender in $87 \%$, and $57.40 \%$ respectively; besides, $90.79 \%$ were newborns at term, $92.93 \%$ had adequate weight for their gestational age and $54.93 \%$ presented neonatal jaundice between 2 and 7 days of extrauterine life. Conclusions: Neonatal jaundice is associated with both modifiable and non-modifiable maternal and neonatal factors that can be addressed with appropriate strategies to reduce the burden of disease.
\end{abstract}

Keywords: Jaundice, neonatal; causality; hyperbilirubinemia; infant, newborn. (Source: DeCS, Bireme). 


\section{Introducción}

Durante siglos la ictericia neonatal se ha observado en los recién nacidos. En 1724, JeanClaude Juncker, en la sinopsis Medicinae Theoretico Praticae, mostró la diferencia entre "ictericia verdadera" y "el tinte ictérico que pueden observarse en los bebés, inmediatamente después de su nacimiento." En 1875, Orth observó durante las autopsias la presencia de la bilirrubina en los ganglios basales de los bebés que tenían ictericia severa, y fue llamada kernicterus por Schmorl en 1903, en 1958, una enfermera del Hospital General de Rothford, Essex, Gran Bretaña, reportó que "la pigmentación amarilla en la piel de los bebés con ictericia se desvanecía cuando habían sido expuestos por cortos periodos de tiempo a la luz del sol"(1).

La ictericia es una de las condiciones más frecuentes en los recién nacidos y una de las principales causas de hospitalización en los servicios de urgencias, debido a que los neonatos en su primera semana de vida, aumentan en menor o mayor grado sus concentraciones séricas de bilirrubinas; esto debido a una destrucción eritrocitaria acelerada, por una insuficiencia hepática transitoria, y por un incremento en la absorción de la circulación enterohepática(2,3).

La ictericia se define como la coloración amarilla de la piel y mucosas, como resultado del incremento en la bilirrubina circulante y se detecta generalmente cuando la bilirrubina sérica total excede los $5 \mathrm{mg} / \mathrm{dl}$. Se clasifica en: ictericia fisiológica, aquella que se produce después del primer día de vida y puede durar hasta una semana y en ictericia patológica que se presenta después de la primera semana de vida y que se produce secundaria a diversas patologías. Aunque esta condición en sus inicios es inofensiva, algunos bebes desarrollan niveles muy altos de bilirrubina y si no se tratan adecuadamente pueden llegar a ser perjudiciales ${ }^{(4)}$.

Cabe destacar que más del $50 \%$ de los recién nacidos desarrollan ictericia, $y$ es de gran importancia conocer que los niveles de bilirrubina que no se normalizan en las primeras dos semanas de vida del recién nacido a término no se pueden considerar como ictericia fisiológica(5).

Entre los factores determinantes que aumentan el riesgo de desarrollar ictericia neonatal, están: prematurez, género masculino, lactancia materna, edad gestacional, cefalohematoma, policitemia, íleo meconial obstructivo, incompatibilidad de grupo y $\mathrm{Rh}$, hipotiroidismo, infecciones del grupo TORCH (toxoplasma, rubéola, citomegalovirus, herpes virus), infecciones el tracto urinario y sepsis; estos factores de riesgo son de gran relevancia y se deben estudiar por el riesgo de complicaciones que pueden comprometer la vida del recién nacido(1,2). El manejo terapéutico de elección para este tipo de hiperbilirrubinemia es la fototerapia, que ayuda a disminuir los niveles de bilirrubina en el recién nacido.

El estudio se efectuó para determinar los factores predisponentes que en gran medida influyen en el desarrollo de la ictericia neonatal en el Hospital Infantil Los Ángeles de Pasto, a través de la revisión de las historias clínicas de los recién nacidos con esta condición. Se utilizó un instrumento diseñado por las investigadoras y avalado por el asesor científico para recolectar la información; el principal limitante de la investigación fue la falta de información en algunas historias clínicas, situación que se corrigió con los criterios de exclusión.

\section{Materiales y métodos}

\section{Tipo de estudio}

Observacional, descriptivo, retrospectivo y cuantitativo.

\section{Selección de la población}

Se trabajó con el total de la población, correspondiente a 684 neonatos egresados del Hospital Infantil Los Ángeles en el periodo enero de 2007- agosto de 2011 con diagnóstico de ictericia neonatal, sin embargo de ellos 76 presentaron criterios de exclusión, los cuales fueron: historias clínicas con datos incompletos, 
letra ilegible o aquellas que no se encontraron en archivo, por lo cual finalmente se analizaron 608 pacientes.

\section{Fuentes de recolección de información}

Este estudio es de tipo retrolectivo pues la información proviene de las historias clínicas, la base de datos del Hospital Infantil Los Ángeles.

\section{Procesamiento de los datos}

Se realizó en Excel, empleando el complemento XLSTAT-Pro 7.5.2.

\section{Técnicas de recolección de información}

Previa autorización del Comité de Ética Médica del Hospital Infantil Los Ángeles para la recolección de información se empleó un instrumento diseñado en su totalidad por las investigadoras, avalado por prueba piloto y revisión del asesor científico. Inicialmente el instrumento estaba constituido por 3 secciones que tenía en cuenta 19 ítems, sin embargo, luego de la realización de la prueba piloto, llevada a cabo con 10 historias clínicas, se eliminaron ítems con información poco útil para el estudio y se añadieron ítems de mayor relevancia para un total de 22, los cuales se dividieron en cuatro secciones, así:

-Datos generales como número de historia clínica, fecha de ingreso y egreso

-Información de la madre como edad, procedencia, hemoclasificación.

-Información del neonato que incluyó edad gestacional, días de vida, género, peso al nacer, hemoclasificación, tipo de alimentación recibida (lactancia materna, fórmula láctea o mixta) -Datos del diagnóstico como patologías asociadas y días de estancia.

\section{Consideraciones éticas}

Según la Resolución 008430 de 1993 del Ministerio de Salud de Colombia, por la cual se establecen las normas científicas, técnicas y administrativas para la investigación en salud, este proyecto está catalogado como investigación sin riesgo, ya que no se realizó ninguna intervención intencional o modificación en las diferentes variables de los individuos que participaron en el estudio, la información se obtuvo de manera retrolectiva a partir de la base de datos e historias clínicas de los pacientes egresados con diagnóstico de ictericia neonatal de la unidad neonatal del Hospital Infantil Los Ángeles, la cual fue suministrada por dicha Institución.

El presente estudio no requirió la realización de consentimiento informado, porque la información provino de la base de datos entregada por el hospital y la revisión de las historias clínicas. La información recolectada se mantuvo bajo custodia, conservando la confidencialidad. Sólo se utilizaron los datos necesarios para los objetivos del proyecto.

\section{Resultados}

El mayor número de casos de ictericia se presentó en hijos de madres jóvenes entre $16 \mathrm{y}$ 20 años con el $30,1 \%$ y entre 21 y 25 años con el $28,5 \%$; la mayoría de las madres procedían del área urbana (74\%) (Tabla 1).

Tabla 1. Características sociodemográficas de las madres de los pacientes con ictericia neonatal

\begin{tabular}{lrr}
\hline Rangos de edad (años) & n & \% \\
\hline $10-15$ & 15 & 2,5 \\
$16-20$ & 183 & 30,1 \\
$21-25$ & 173 & 28,5 \\
$26-30$ & 101 & 16,6 \\
$31-35$ & 76 & 12,5 \\
$36-40$ & 32 & 5,3 \\
$41-45$ & 8 & 1,3 \\
Sin dato & 20 & 3,3 \\
Área de procedencia & & \\
Rural & 161 & 26 \\
Urbana & 447 & 74 \\
\hline
\end{tabular}

El $90,79 \%$ de neonatos con ictericia fueron recién nacidos a término y en menor porcentaje recién nacidos pretérmino y postérmino con $7,73 \%$ y $1,48 \%$, respectivamente. Con respecto al peso para la edad gestacional, 92,93\% tuvieron un peso adecuado, 5,59\% bajo peso y 1,48\% fueron grandes para la edad gestacional. En cuanto a la incompatibilidad de grupo sanguíneo y de $\mathrm{Rh}, 21,22 \%$ presentaron incompatibilidad 
ABO, de tipo A fue la más frecuente de ese grupo con un $63,57 \%$ y el $2,96 \%$ del total presentaron incompatibilidad Rh. De los pacientes estudiados el $87 \%$ recibieron lactancia materna exclusiva, $11 \%$ fórmula láctea o lactancia mixta (leche materna + fórmula láctea) y en $2 \%$ no se encontró la información (Tabla 2).

Tabla 2. Características basales de los pacientes con ictericia neonatal egresados de la UCI neonatal

\begin{tabular}{lrr}
\hline & Fr & \multicolumn{1}{c}{$\mathbf{\%}$} \\
\hline Clasificación edad gestacional & & \\
Pretérmino & 47 & 7,73 \\
Término & 552 & 90,79 \\
Postérmino & 9 & 1,48 \\
Clasificación peso al nacer & & \\
Bajo para la edad gestacional & 34 & 5,59 \\
Adecuado para la edad & 565 & 92,93 \\
gestacional & & \\
Grande para la edad gestacional & 9 & 1,48 \\
Incompatibilidad ABo & $\mathbf{1 2 9}$ & $\mathbf{2 1 , 2 2}$ \\
A & 82 & 13,40 \\
B & 46 & 7,50 \\
AB & 1 & 0,16 \\
Incompatibilidad Rh & $\mathbf{1 8}$ & $\mathbf{2 , 9 6}$ \\
Alimentación & & \\
Lactancia materna exclusiva & 530 & 87,00 \\
Fórmula láctea/Lactancia mixta & 66 & 11,00 \\
Sin información & 12 & 2,00 \\
\hline
\end{tabular}

En la Tabla 3 se observa que la ictericia neonatal, se presentó con mayor frecuencia en el género masculino y entre los 2 y 7 días de vida extrauterina, con un porcentaje del 57,4\% y $54,93 \%$ respectivamente.

Tabla 3. Pacientes con ictericia neonatal egresados de la UCI neonatal, según género y edad en el momento

\begin{tabular}{ccccccl}
\hline $\begin{array}{c}\text { Género } \\
\text { Edad }\end{array}$ & \multicolumn{2}{c}{ Femenino } & \multicolumn{2}{c}{ Masculino } & Total & \% \\
\hline 24 horas & 17 & 2,80 & 18 & 2,96 & 35 & 5,76 \\
2-7 días & 146 & 24,01 & 188 & 30,92 & 334 & 54,93 \\
8-14 días & 74 & 12,17 & 100 & 16,45 & 174 & 28,62 \\
15-21 días & 18 & 2,96 & 32 & 5,26 & 50 & 8,22 \\
22-28 días & 4 & 0,66 & 11 & 1,81 & 15 & 2,47 \\
Total & $\mathbf{2 5 9}$ & $\mathbf{4 2 , 6 0}$ & $\mathbf{3 4 9}$ & $\mathbf{5 7 , 4 0}$ & $\mathbf{6 0 8}$ & $\mathbf{1 0 0}$ \\
\hline
\end{tabular}

De las comorbilidades, la que conlleva a mayor gravedad, es la sepsis neonatal, que se presentó en el $5,26 \%$ de pacientes conjuntamente con ictericia neonatal y de otras patologías en un $14,80 \%$, siendo la más frecuente la onfalitis en un $3,95 \%$ (Tabla 4 ).

Tabla 4. Comorbilidades Neonatales de pacientes egresados de la UCI neonatal

\begin{tabular}{lrr}
\hline \multicolumn{1}{c}{ Comorbilidad } & n & $\mathbf{0}$ \\
\hline Sepsis neonatal & $\mathbf{3 2}$ & $\mathbf{5 , 2 6}$ \\
Otras patologías & $\mathbf{9 0}$ & $\mathbf{1 4 , 8}$ \\
Onfalitis & 24 & 3,95 \\
Cefalohematoma & 18 & 2,96 \\
Enfermedad diarreica & 14 & 2,30 \\
Policitemia & 11 & 1,81 \\
Otras infecciones & 11 & 1,81 \\
Anemia por hemólisis & 7 & 1,15 \\
Hipotiroidismo neonatal & 4 & 0,66 \\
Colestasis neonatal & 1 & 0,16 \\
Ninguna & 518 & 85,20 \\
\hline
\end{tabular}

\section{Discusión}

La hiperbilirrubinemia neonatal está asociada con factores tanto de la madre como del neonato, y podrían ser intervenidos efectivamente para reducir la carga de enfermedad en países de bajos y medianos ingresos(6). En el presente estudio se encontró que las madres de los recién nacidos con ictericia predominantemente eran madres jóvenes entre los 16 y 20 años (30,1\%), lo cual se asemeja al estudio realizado por Riscarle et al., donde se determinó que la mayoría de en partos en adolescentes de 17 a 19 años de edad el 57,40\% y 38,20\% de sus recién nacidos manifestaron patologías perinatales, entre las más frecuentes, la ictericia neonatal(7). De igual manera, en un estudio llevado a cabo en 6 países de bajos y medianos ingresos (Kenia, Zambia, India, Pakistán, Guatemala y Argentina), el porcentaje predominante de embarazos se presentó en mujeres entre 15 y 19 años, evidenciando una mayor morbilidad perinatal con respecto a otros grupos ${ }^{(8)}$.

Es de notar que $73,52 \%$ de los casos con esta patología en el Hospital Infantil Los Ángeles, fueron hijos de madres de procedencia urbana, lo cual podría deberse a la mayor posibilidad de acceso a la consulta con respecto a la población del área rural, ya que de acuerdo a los indicadores básicos de salud del 2014 en Colombia para el año 2007 el 11,29\% de la 
población no se encontraba afiliada a seguridad social en salud, lo cual disminuyó al 8,33\% en el 2011(9), mas no indica directamente que la procedencia sea un factor predisponente para ictericia en los neonatos.

La ictericia neonatal se presenta comúnmente en los recién nacidos cerca del término y el $80 \%$ de los pretérmino(10), en el estudio de Rojas et al., esta condición estuvo presente en el $61,70 \%$ de los neonatos pretérmino(11); sin embargo, estos datos no se correlacionan con la presente investigación debido a que la población en el período descrito solo incluyó a 47 prematuros de 608 neonatos estudiados.

Un estudio realizado en el hospital de Namazi en Irán por Najib et al., reportó que el 58,20\% de los pacientes con ictericia neonatal fue del género masculino(12), similar al realizado en Nigeria por Onyearugha Onyire et al., con un $55,40 \%{ }^{(13)}$ y otro llevado a cabo en los hospitales de Qods y Kowsar en Qazvin en 2014, donde el 53,30\% de los neonatos con ictericia pertenecieron al género masculino(14), esto es comparable con el actual estudio, ya que el $57,4 \%$ de los pacientes fueron hombres, aunque las razones son desconocidas, varios estudios han informado un riesgo incrementado de ictericia neonatal grave en el género masculino comparado con el femenino, la mayor prevalencia del síndrome de Gilbert y la deficiencia de glucosa-6-fosfato deshidrogenasa (G6PD) en los varones, explican parcialmente la mayor incidencia de ictericia neonatal grave y la susceptibilidad de la lesión inducida por bilirrubina(15).

La hiperbilirrubinemia neonatal es un problema clínico común encontrado durante este periodo, especialmente en la primera semana de vida, la ictericia fisiológica es el tipo más frecuente, presentándose entre las 24 a 72 horas posteriores al nacimiento(16). Cercano a los hallazgos de esta investigación, donde la ictericia neonatal se manifestó entre el segundo y séptimo día de vida extrauterina en un 54,93\%, González et al(17), encontraron en su estudio que el $76,87 \%$ de los neonatos presentaron ictericia después de las 48 horas de vida, lo cual podría ser explicado por el incremento en la carga de bilirrubina por mayor volumen de glóbulos rojos y disminución de la supervivencia de los mismos, con aumento de la eritropoyesis ineficaz; aumento de la circulación enterohepática, inmadurez enzimática por disminución de la actividad de la uridinadifosfato glucuroniltransferasa (UDPGT), captación defectuosa de la bilirrubina provocado por la menor cantidad de ligandinas y reducción de la excreción hepática de bilirrubina(18).

En esta investigación se encontró que el $87 \%$ de los neonatos fueron alimentados exclusivamente con lactancia materna, comparable a un estudio realizado en el hospital II Ramón Castilla de Lima, donde el 93,34\% de los pacientes recibieron lactancia materna exclusiva(19), factor de riesgo conocido para presentar hiperbilirrubinemia pero con mayores beneficios que posibles daños(20) y un mecanismo de asociación aún no bien entendido(21), aunque se sugiere puede deberse a ingesta reducida, inhibición de la excreción hepática de bilirrubina por inhibición de la enzima UDPGT, secundaria a la presencia de varias sustancias en la leche materna como pregnane $3,20 \beta$-diol, ácidos grasos libres, lipasas, iones metálicos, esteroides y nucleótidos, aumento en la reabsorción de bilirrubinas a nivel intestinal, disminución de la eliminación de meconio y alteraciones en el metabolismo de los ácidos biliares(22,23); cabe aclarar que en un porcentaje no despreciable de los pacientes mencionados en el presente estudio, existían condiciones concomitantes además de la lactancia materna exclusiva, la incompatibilidad de grupo o $\mathrm{Rh}$, infecciones, prematuridad, entre otras.

En la población estudiada se identificó además que la ictericia neonatal fue secundaria a incompatibilidad $\mathrm{ABO}$ en un $21,22 \%$ de los casos, de los cuales, $63,5 \%$ fueron incompatibilidad AO, hechos que son apoyados por el estudio realizado en una unidad neonatal de un Hospital Pakistaní, donde $32,40 \%$ de los neonatos con ictericia mostraron incompatibilidad de grupo(24), y un reporte de la revista Blood, en el cual la incompatibilidad AO se presentó en un $57,8 \%$ de los pacientes $(25)$. 
Las infecciones bacterianas fue otro factor relacionado con ictericia neonatal en la presente investigación, resultado semejante a un estudio realizado en un Hospital de Irán por Maamouri et al., donde concluyeron que la infección bacteriana fue una causa significativa de hiperbilirrubinemia inexplicada entre los recién nacidos con presencia de ictericia en un $10 \%$ de los pacientes(26). Es importante resaltar que en el $85,20 \%$ de los pacientes estudiados, no hubo comorbilidades identificables, lo que confirma que el principal tipo de ictericia neonatal es fisiológica, como se explicó previamente.

Por último, es necesario recordar que la deficiencia de G6PD es el defecto enzimático humano más frecuente, estimándose en 400 millones de individuos en todo el mundo, la incidencia de ictericia neonatal es varias veces mayor en los niños deficientes de esta enzima en comparación con los que tienen niveles normales de la misma(27). En un estudio realizado en el departamento de pediatría de un complejo médico en Baréin, se determinó que de los pacientes estudiados con ictericia neonatal, el $42 \%$ presentaban deficiencia de la enzima G6PD y en su mayoría eran varones (28), teniendo en cuenta esta información y ya que no hay publicaciones sobre esta deficiencia en la población pediátrica Nariñense sería pertinente realizar estudios acerca de su prevalencia y las complicaciones que puede ocasionar.

\section{Conclusiones}

A pesar de que hay pocas investigaciones sobre ictericia neonatal en la población Nariñense, el estudio mostró que algunos factores predisponentes para dicha afección son: la edad materna, en el rango de adolescencia tardía (1620 años) y la sepsis neonatal. Teniendo en cuenta esta información, a través de estrategias de promoción y prevención es posible evitar el embarazo en adolescentes e intervenir sobre los factores de riesgo para sepsis neonatal en todos los niveles de atención mediante los programas de consulta preconcepcional, control prenatal, atención del parto y puerperio y atención del recién nacido, de tal manera que se disminuya el desarrollo de hiperbilirrubinemia neonatal.
Sin embargo, otros factores predisponentes encontrados, como el género masculino y la incompatibilidad de grupo $\mathrm{ABO}$, no son modificables, pero es relevante conocerlos para realizar un manejo oportuno y prevenir así complicaciones.

Finalmente, con la presente investigación, se hace evidente la necesidad de realizar otros estudios en el departamento de Nariño, acerca de la relación entre la lactancia materna y la ictericia neonatal, dado que la alimentación con leche materna exclusiva fue una condición presente en un gran número de los casos estudiados.

\section{Conflicto de intereses}

Los autores declaran que no existe conflicto de intereses.

\section{Referencias}

1. Lauer BJ, Spector ND. Hyperbilirubinemia in the Newborn. Pediatr Rev. 2011;32(8):341-9.

2. Gallegos-Dávila JA, Rodríguez-Balderrama I, RodríguezBonito R, Abrego-Moya V, Rodríguez-Camelo G. Prevalencia y factores de riesgo para hiperbilirrubinemia indirecta neonatal en un hospital universitario. Med Univ. 2009;11(45):226-30.

3. Wong RJ, Rand EB, Kim MS. Pathogenesis and Etiology of Unconjugated Hyperbillirubinemia in the newborn. UpToDate. 2015;(figure 1):1-10.

4. National Institute for Health and Excellence. Neonatal jaundice. MidCity Place. 2010;5(May):457-75.

5. Omeñaca Teres F, González Gallardo M. Ictericia neonatal. Pediatr Integr. 2015;18(6):367-74.

6. Olusanya BO, Osibanjo FB, Slusher TM. Risk factors for severe neonatal hyperbilirubinemia in low and middleincome countries: A systematic review and metaanalysis. PLoS One. 2015;10(2):1-16.

7. Manrique R, Rivero A, Ortunio M, Rivas M, Cardozo Castellano de A R, Guevara Rivas H. Parto pretérmino en adolescentes. Revista de Obstetricia y Ginecología de Venezuela. 2008;68:141-143.

8. Althabe F, Moore JL, Gibbons L, Berrueta M, Goudar SS, Chomba E, et al. Adverse maternal and perinatal outcomes in adolescent pregnancies: The Global Network's Maternal Newborn Health Registry study. Reprod Health. Bio Med Central. 2015;12(Suppl 2):S8S8.

9. Ministerio de Salud y Protección Social. Indicadores Básicos 2014. Situación de Salud en Colombia [Internet]. 2015;1-140. Available from: https://www.minsalud.gov.co/sites/rid/Lists/Bibliote caDigital/RIDE/VS/ED/PSP/Indicadores-basicossalud-2014.pdf 
10. Scrafford CG, Mullany LC, Katz J, Khatry SK, Leclerq SC, Darmstadt GL, et al. Incidence of and risk factors for neonatal jaundice among newborns in southern Nepal. Trop Med Int Heal. 2013;18(11):1317-28.

11. Rojas Feria P, Pavón Delgado A, Rosso González M, Losada Martínez A. Complicaciones a corto plazo de los recién nacidos pretérmino tardíos. An Pediatría. Elsevier; 2011 Sep 1;75(3):169-74.

12. Najib KS, Saki F, Hemmati F, Inaloo S. Incidence, Risk Factors and Causes of Severe Neonatal Hyperbilirubinemia in the South of Iran (Fars Province). Iran Red Crescent Med J. 2013;15(3):260-3.

13. Onyearugha $\mathrm{CN}$, Onyire BN, Ugboma HAA. Neonatal jaundice: Prevalence and associated factors as seen in Federal Medical Centre Abakaliki, Southeast Nigeria. J Clin Med Res. 2011;3(3):40-5.

14. Garosi E, Mohammadi F, Ranjkesh F. The relationship between neonatal jaundice and maternal and neonatal factors. Iran J Neonatol. 2016;7(1):37-40.

15. Sachdeva A, Dutta AK. Advances in Pediatrics. 2 ed. New Delhi, Panamá, London: Jaypee Brothers Medical Publishers (P). Ltd; 2012. Disponible en: https://books.google.com.co/books?id=I2FHFyCaDeIC

16. Ullah S, Rahman K, Hedayati M. Hyperbilirubinemia in neonates: Types, causes, clinical examinations, preventive measures and treatments: A narrative review article. Iranian Journal of Public Health. 2016;45(5):558-68.

17. González AC, Uría RMA, Morán RA, López IB, Aguilar RD, Pérez MR. Hiperbilirrubinemia neonatal agravada. Rev Cubana Pediatr. 2010;82(3):13-9.

18. Martínez de la Barrera LI. Ictericia neonatalHiperbilirrubinemia indirecta. Precop SCP [Internet]. 2012;12(2):38-45.

19. Carrasco-Tejerina SH. Prevalencia de Ictericia Neonatal, (Hiperbilirrubinemia intermedia) y factores asociados en Recién nacidos a término en el Hospital II Ramón Castilla - EsSalud durante el año 2014. [Tesis pregrado] Lima, Perú: Universidad Ricardo Palma; 2016. Disponible en: http://cybertesis.urp.edu.pe/bitstream/urp/480/1/Ca rrasco_s.pdf

20. Sánchez-Redondo Sánchez-Gabriel MD, Leante Castellanos JL, Benavente Fernández I, Pérez Muñuzuri A, Rite Gracia S, Ruiz Campillo CW, et al. Recomendaciones para la prevención, la detección y el manejo de la hiperbilirrubinemia en los recién nacidos con 35 o más semanas de edad gestacional. An Pediatría [Internet]. 2017; Available from: http://www.sciencedirect.com/science/article/pii/S16 95403317301510

21. Olusanya BO, Slusher TM. Infants at risk of significant hyperbilirubinemia in poorly-resourced countries: evidence from a scoping review. World J Pediatr. 2015;11(4):293-9.

22. Chou HC, Chen MH, Yang HI, Su YN, Hsieh WS, Chen CY, et al. $211 \mathrm{G}$ to $\mathrm{A}$ variation of UDP-glucuronosyl transferase $1 \mathrm{~A} 1$ gene and neonatal Breastfeeding jaundice. Pediatr Res. 2011;69(2):170-4.

23. Fujiwara R, Maruo $Y$, Chen S, Tukey RH. Role of extrahepatic UDP-glucuronosyltransferase 1A1:
Advances in understanding breast milk-induced neonatal hyperbilirubinemia. Toxicol Appl Pharmacol. 2015 Nov;289(1):124-32.

24. Hussain M, Irshad M, Kalim M, Ali L, Ali L. Glucose-6phosphate dehydrogenase deficiency in jaundiced neonates. JPMI - J Postgrad Med Inst. 2010;24(2):121-6.

25. Rao LB, Ahmed Z, Ozgonenel B. The Clinical Spectrum of ABO Incompatibility and Hemolytic Disease in the Newborn. Blood. 2015;120(21):1182.

26. Maamouri G, Khatami F, Mohammadzadeh A. Hyperbilirubinemia and Neonatal Infection. International Journal of Pediatrics. 2013;1(1):5-12.

27. Bhutani VK. Jaundice Due to Glucose-6-Phosphate Dehydrogenase Deficiency. Neoreviews. 2012;13(3):e166-77.

28. Isa HM, Mohamed MS, Mohamed AM, Abdulla A, Abdulla F. Neonatal indirect hyperbilirubinemia and glucose-6-phosphate dehydrogenase deficiency. Korean J Pediatr. 2017;60(4):106-11. 